\title{
PERSPECTIVE
}

\section{Strategies for the management of microbial keratitis}

\author{
Bruce D S Allan, John K G Dart
}

Microbial keratitis results from a complex interaction between a protean array of pathogens and a diversity of host responses. The variability of these factors has hampered the development of simple management guidelines that will ensure an optimal outcome for most cases; a consequence of this has been the use of an extensive range of culture media in initial investigation, toxic levels of antibiotic treatment, and constant clinical review, all of which may be unnecessary. The simplified initial management strategies described here are designed to provide effective treatment for the majority of cases, and to identify the point of failure when referral for specialist management may be indicated. A systematic approach to the further management of cases in which initial therapy has failed is also given. These recommendations have been developed to be both practical and safe.

\section{Presentation}

Microbial keratitis is rare in the absence of predisposing factors. ${ }^{12}$ Prominent risk factors include: contact lens

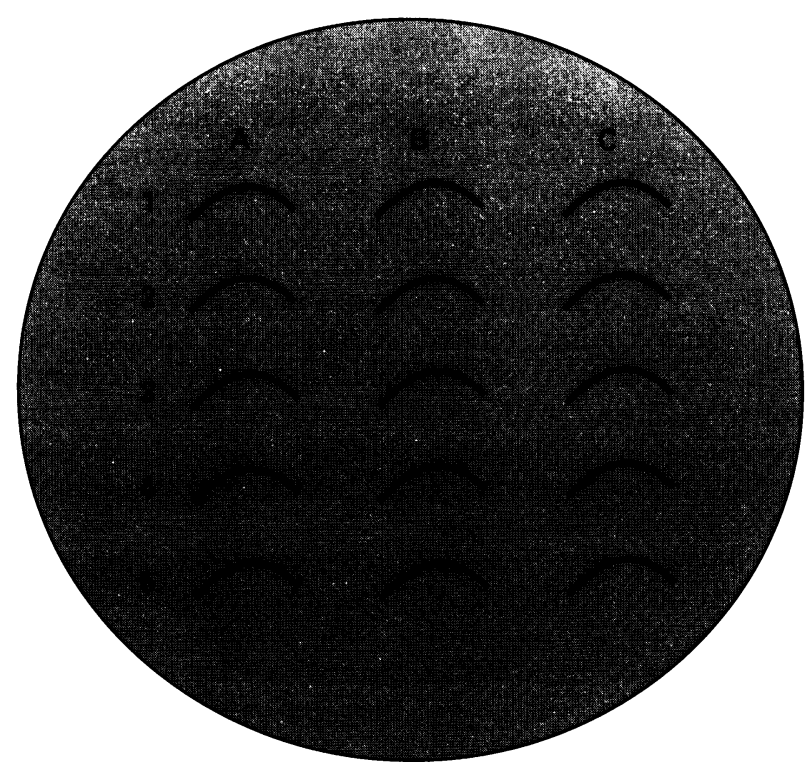

Figure 1 A preferred pattern ${ }^{8}$ for inoculating solid media is illustrated schematically above. Each scraping is wiped across the media surface in a column of five or six curvilinear streaks. Columns $A$ to $C$ each represent $a$ separate inoculum. Rows 1-5 represent successive applications of the same debrided material. Corneal scraping is normally performed using unpreserved topical anaesthesia (preservatives may interfere with bacterial growth in culture). Slough and debris are cleared from the ulcer surface. A 21 gauge needle is then used to debride the ulcer edge or the ulcer base (purulent debris is less likely to contain live organisms than tissue from the interface between visibly involved and normal stroma). Debrided material is spread in a thin layer over slides, which may be left to air dry. Further scrapings are used to inoculate culture media. Care should be taken not to break the gel surface when inoculating agar plates. Pending examination by a microbiologist, slides and the patient's contact lens cases and cleaning solutions, if available, should be refrigerated $\left(4^{\circ} \mathrm{C}\right)$. Agar plates (and any liquid media) should be placed in an incubator $\left(35-37^{\circ} \mathrm{C}\right)$. wear ${ }^{3}$ (especially overnight wear), ${ }^{4}$ trauma, and ocular surface compromise (for example, herpes simplex keratitis, exposure, corneal anaesthesia, bullous keratopathy, etc).

Infection should be anticipated in epithelial defects in immunocompromised patients or those using topical steroid medication. Corneal infiltration by leucocytes, indicating the presence of infection, is inhibited in these situations, and microbial keratitis can present as a nonhealing epithelial defect or an exacerbation of a stromal melting condition. Conversely, bacterial keratitis (but not amoebic keratitis) is unusual in the absence of a corneal epithelial defect. Multifocal peripheral infiltrates in contact lens wearers or marginal infiltrates without an overlying epithelial defect are usually a sterile immunological reaction to bacterial antigen deposition in association with contact lens case or lid margin colonisation. ${ }^{56}$

\section{Initial investigation}

Corneal scraping (Fig 1), for diagnostic cultures, and intensive topical antibiotic treatment are indicated wherever microbial keratitis is suspected. ${ }^{78}$ Beyond its diagnostic value, scraping may accelerate disease resolution by enhancing antibiotic penetration ${ }^{9}$ and the therapeutic debridement of necrotic tissue. Baseline indices of disease severity should probably be recorded after, rather than before, scraping (Fig 2). Confusion may otherwise arise in relation to the size of the corneal epithelial defect during subsequent monitoring of disease progression. No reports exist of endophthalmitis occurring in bacterial keratitis without perforation. Anterior chamber and vitreous taps may introduce infection and are not normally helpful where perforation is absent.

Pre-prepared scraping and treatment kits should ideally be available in the emergency room. Routine scraping kits

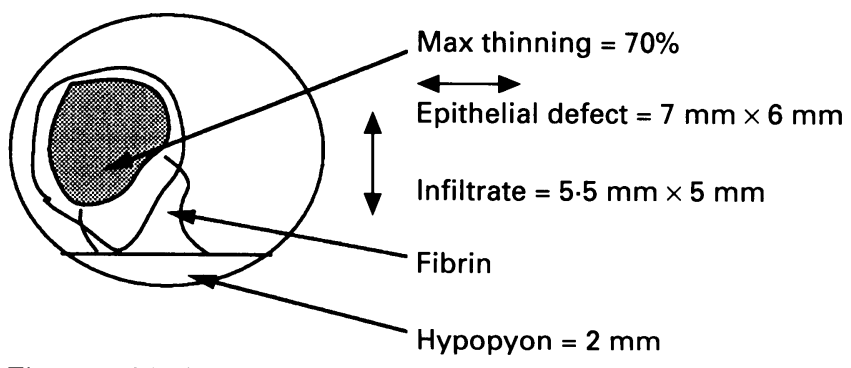

Figure 2 The following indices of disease severity are normally recorded: (1) the dimensions of the lesion - recording the maximum length and the width of the epithelial defect and the subjacent infiltrate in two orthogonally placed axes with an indication of their orientation; (2) an estimate of the maximum stromal thinning - expressed as a percentage of normal corneal thickness, together with an indication of the location of maximal thinning within the lesion; (3) the height of any hypopyon, and the extent of any anterior chamber fibrin deposition and cellular reaction. Pain is an important subjective index of disease progression. Baseline examination should also include a full assessment of ocular surface integrity with special consideration of factors such as lid function, the tear film, and corneal sensation. 
Table 1 Presenting features of fungal and acanthamoebal keratitis

\begin{tabular}{lll}
\hline & History & Examination \\
\hline Fungi $^{13}$ & $\begin{array}{c}\text { Trauma (esp vegetable foreign } \\
\text { body) } \\
\text { Chronic ocular surface disease } \\
\text { (Candida infection) }\end{array}$ & $\begin{array}{l}\text { Infiltrate with feathery } \\
\text { margins }\end{array}$ \\
& $\begin{array}{l}\text { Tropical travel } \\
\text { Tcanthamoeba }{ }^{14}\end{array}$ & $\begin{array}{l}\text { Contact lens wear } \\
\text { Dry, rough surface texture }\end{array}$ \\
& $\begin{array}{c}\text { Corneal trauma with fresh } \\
\text { water or soil contamination }\end{array}$ & $\begin{array}{l}\text { Epitheliopathy (disc, ring, } \\
\text { or dendriform) }\end{array}$ \\
& $\begin{array}{l}\text { Pain out of proportion with } \\
\text { subtle examination findings }\end{array}$ & $\begin{array}{l}\text { Perineural infiltrates } \\
\text { Low grade anterior uveitis }\end{array}$ \\
& $\begin{array}{c}\text { Misdiagnosis of herpes } \\
\text { simplex keratitis }\end{array}$ & \\
\hline
\end{tabular}

should include, at minimum, one slide for Gram staining, and one agar plate for aerobic incubation. Laboratory procedures in ocular microbiology are tailored to the small amount of material and small numbers of organisms obtainable from the eye; it is important to inoculate ocular specimens directly onto the media and avoid the use of transport or storage media. Some of the pathogens isolated from ocular infections may be considered to be normal flora by non-ocular microbiologists. It is important that ophthalmologists liaise with the laboratory to make these requirements known. ${ }^{8}$ The vast majority of isolates from microbial keratitis in temperate climates (for example, London, ${ }^{10}$ Boston, $^{2}$ or Adelaide ${ }^{1}$ ) are aerobic bacteria. Almost all of these, and most fungi, can be cultured successfully on blood agar. ${ }^{11}$ Additional slides for specialised stains and a variety of other solid and liquid culture media are useful in identifying some less common pathogens, ${ }^{12}$ but their routine use in the absence of clinical and epidemiological features suggestive of fungal, ${ }^{13}$ or amoebic infection ${ }^{14}$ (Table 1 ) is probably unnecessary, particularly where access to material is limited in small ulcers or with impending perforation. The premise here is that organisms that do not multiply easily on simple media in aerobic conditions will not multiply easily in the cornea either, and are unlikely to produce rapidly progressive infection. While the inoculation of a comprehensive array of media before primary therapy may be preferable where this is practical, the initiation of treatment should not be delayed if additional culture media are unavailable. ${ }^{15}$

Routine microscopy of smears is useful to help screen out fungal infections and corroborate later culture results. However, because polymicrobial infections are relatively common, ${ }^{12710}$ and a negative Gram stain does not exclude bacterial infection, ${ }^{16}$ initial therapy will usually be intensive broad spectrum topical antibacterial treatment regardless of the Gram stain findings.

\section{Initial therapy: a simplified two phase approach}

There have been two different approaches to the initial treatment of microbial keratitis: 'shotgun therapy', in which the choice of a combination of fortified antibiotics, used in intensive initial therapy, is based on local epidemiological information regarding likely infecting organisms ${ }^{15}$; and specific therapy, in which intensive treatment with a single antibiotic is directed by the results of the microbiological investigation. ${ }^{17}$ Each has disadvantages. Complete antibiotic cover is not possible, and treatment toxicity is more likely with shotgun therapy; whereas specific therapy risks disease progression if microbiological investigations are incomplete or misleading. A compromise approach is often adopted in which shotgun therapy is continued until clear evidence of the infecting agent and its antimicrobial sensitivities emerges to direct specific therapy. ${ }^{7}$ This, however, leaves a number of potential areas of confusion. Recommendations with regard to the frequency of follow up and the duration of intensive therapy, for example, have been vague. The administration frequency of topical antibiotics is often tailed off gradually as inflammation resolves $^{7}$; but there is no clear logical basis for this practice.

Clinical review is, at best, only a loose guide to the point at which the cornea has been sterilised after the initiation of intensive therapy. ${ }^{7}$ Sterilisation almost certainly precedes epithelial healing and the resolution of inflammatory signs; and preservative or agent related toxicity may delay epithelial healing where intensive topical treatment is prolonged.

Antibiotic treatment in this context is designed either to sterilise the cornea (intensive therapy) or to prevent superinfection (prophylaxis). We would suggest that initial therapy in microbial keratitis may be divided into two distinct phases with clearly defined points for clinical review and decision making: a limited period of intensive antibiotic treatment designed to sterilise the cornea (phase 1 - sterilisation) (Fig 3); and a second period in which therapy is aimed at limiting further inflammatory damage, preventing superinfection, and promoting epithelial healing (phase 2 - healing) (Fig 4).

\section{Antibiotic choice}

Trials of combination therapy with fortified aminoglycoside (for example, gentamicin) and second generation cephalosporin (for example, cefuroxime) antibiotics versus monotherapy (single antibiotic treatment) with new generation fluoroquinolones (for example, ofloxacin or ciprofloxacin that are commercially available in topical preparations suitable for treating keratitis) are still in progress. While both approaches are widely used and offer good broad spectrum cover against the majority of likely isolates in a temperate climate, the choice of primary therapy should ultimately be governed by contemporary local epidemiological findings. ${ }^{15}$ Monotherapy with a fluoroquinolone antibiotic may be most appropriate in the developed world where contact lens related pseudomonal keratitis is increasingly predominant ${ }^{3} 10$ and pseudomonal resistance to aminoglycosides has become a significant problem. ${ }^{18}$ In developing countries such as Nepal, however, where trauma related streptococcal keratitis predominates, ${ }^{19}$ a form of combined therapy (for example, fortified penicillin with ofloxacin) may be preferable since fluoroquinolones, although clinically effective in many cases of streptococcal keratitis, ${ }^{2021}$ are generally less active against streptococcal species. ${ }^{22}$

A clinical trial with an $80 \%$ chance of detecting a $5 \%$ difference in the efficacy of these treatment regimens, if this exists, would require over 1000 patients. This power analysis is based on published figures showing success after initial therapy in over $90 \%$ of cases for ciprofloxacin and a similar figure for conventional combination therapy. ${ }^{20}$ Definitive evidence on which to base antibiotic choice is therefore unlikely to be available in the near future, if at all, given that resistance patterns to new antibiotic regimens will probably continue to change.

Fluoroquinalones are less toxic than aminoglycosides 2324 which are not readily available as fortified preparations. Monotherapy may facilitate compliance and eliminates dilutional effects where different drops are administered simultaneously. Provided that there is broad parity between the efficacy of treatment with standard preparations of fluoroquinalone antibiotics and fortified combination therapy, and the emerging view is that there is, ${ }^{2021}$ it is reasonable and practical to recommend monotherapy 
Initial therapy-phase 1-sterilisation

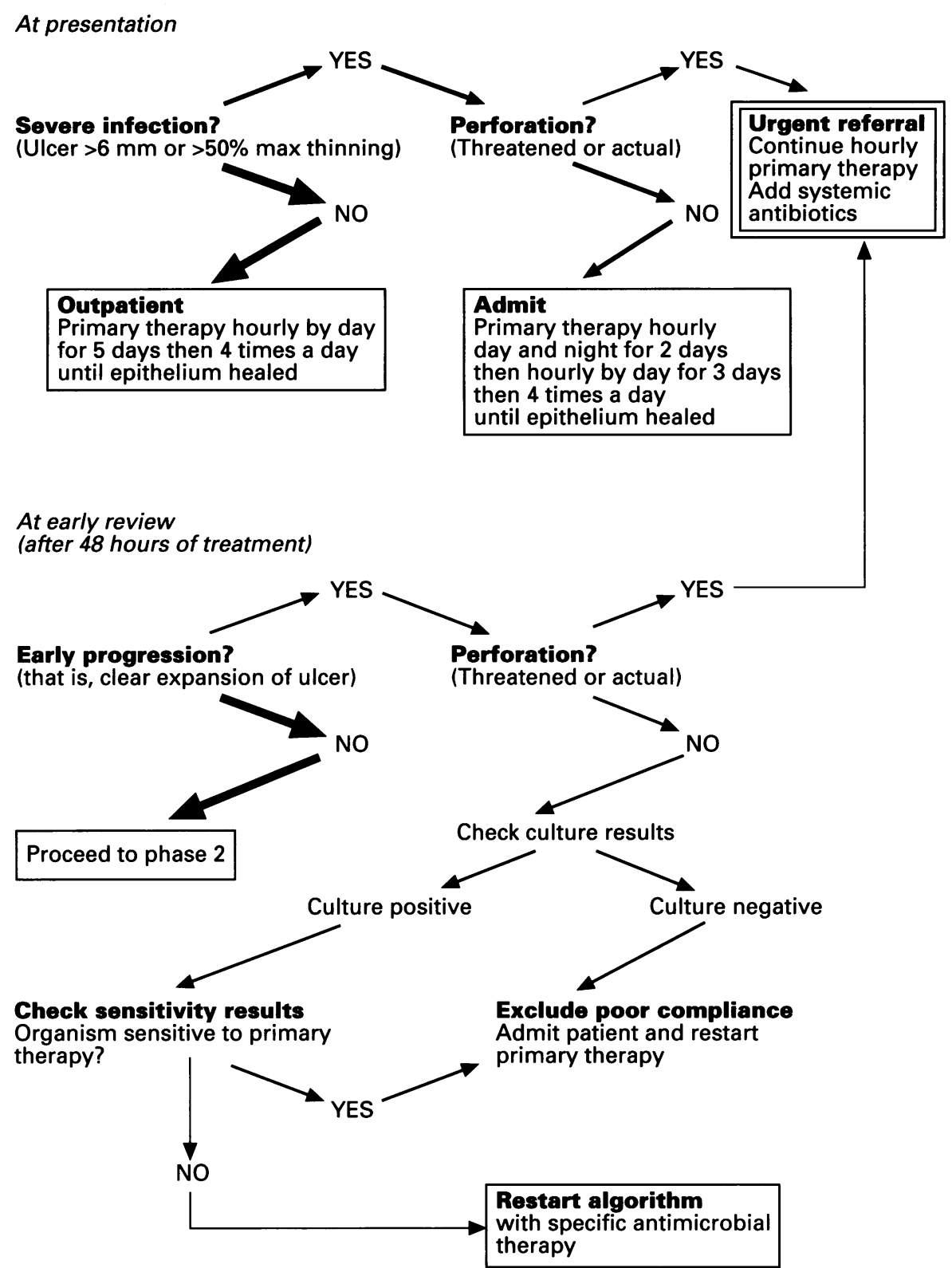

with a fluoroquinolone preparation as the initial broad spectrum antibiotic treatment for microbial keratitis in the United Kingdom. Fluoroquinalones available for topical ophthalmic use are norfloxacin, ofloxacin, and ciprofloxacin, all as $0.3 \%$ solutions. Clinical trials of these in keratitis are only available for ciprofloxacin $0.3 \%$ and have shown similar ${ }^{20}$ or better results ${ }^{21}$ than for conventional therapy in controlled but not randomised studies. In vitro antimicrobial sensitivity studies show that norfloxacin is less active against Gram positive isolates than either of the other two quinolones for non-ocular isolates ${ }^{22}$ and this has been confirmed for ocular isolates in comparison with ofloxacin. ${ }^{25}$ For these reasons norfloxacin is the least appropriate of these available quinolones as broad spectrum monotherapy for bacterial keratitis. Either ciprofloxacin 2021 or ofloxacin should be effective as monotherapy for most cases of bacterial keratitis. Although the results of clinical trials are not yet available for ofloxacin in the management of keratitis it penetrates the cornea better than other commercially available fluoroquinolones $^{9}$ and human tear film concentrations ${ }^{26}$ of ofloxacin, 4 hours after topical administration (9.16 (SD $8.24) \mathrm{mg} / \mathrm{l})$, exceed the $\mathrm{MIC}_{90}(2 \mu \mathrm{g} / \mathrm{ml})$ for a wide range of ocular isolates. ${ }^{25}$ Ofloxacin, unlike ciprofloxacin, does not induce corneal plaques. ${ }^{20} 2127$ These data suggest that ofloxacin should be as effective as ciprofloxacin for bacterial keratitis and further studies will clarify the role of these drugs in streptococcal keratitis and the optimal dosage schedules.

Phase one - sterilisation

Although it is probably impossible to specify a dose frequency and duration for intensive topical antibiotic therapy which will always guarantee sterilisation, hourly administration for 5 days (Fig 3) should leave a wide margin of safety. Initial results obtained with this defined period of intensive broad spectrum therapy (unpublished 
Initial therapy-phase 2-healing

At review after 1 week of therapy

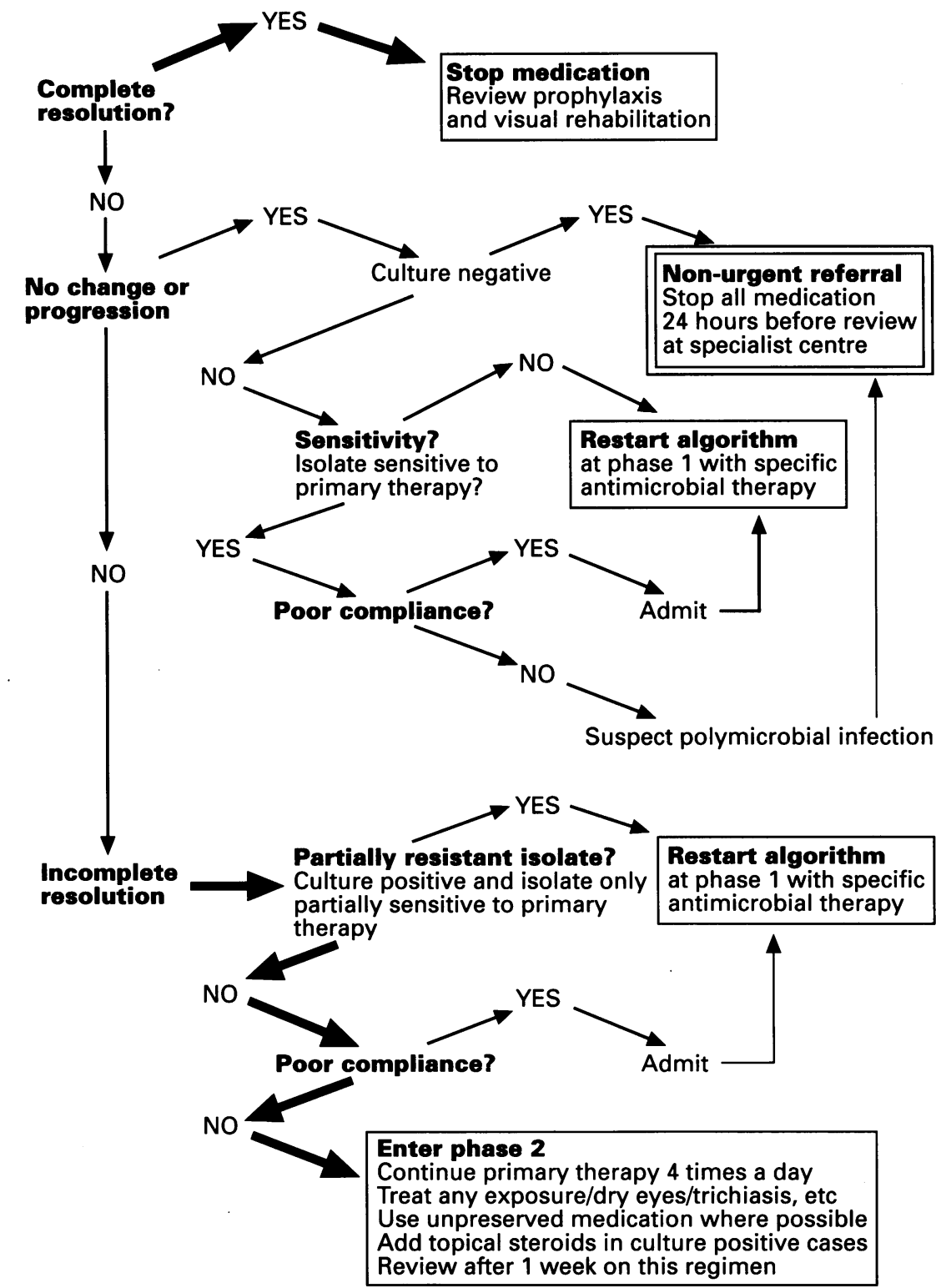

Figure 4 Strategies for patient management at review around 1 week after presentation are summarised in this flow chart. Again bold arrows denote relative proportions of patients following each route. In a review of consecutive patients $(n=70)$ treated in this department in 1993/4 (unpublished data), using either ofloxacin or fortified combination treatment (cefuroxime and gentamicin) as intensive initial therapy, approximately two thirds of patients had healed at 1 week and over 90\% had healed within 1 month (that is, after up to 3 weeks of phase 2 therapy).

data - Fig 4) compare well with those for contemporary studies in which high dose antibiotic treatment was gradually reduced..$^{20} 21$

The majority of patients with microbial keratitis can be managed successfully as outpatients ${ }^{28}$ with initial review after 48 hours. Admission may be necessary where good compliance is unlikely (for example, elderly or debilitated patients with poor social support); or for overnight treatment in severe infections (axial lesions, lesions $6 \mathrm{~mm}$ or more in diameter, or with $50 \%$ or more stromal thinning) in which the margin for error in terms of undertreatment or poor compliance may be smaller. The general value of overnight treatment may be open to question, given that tear film quinolone concentrations are so well maintained.

Systemic antibiotics (for example, ciprofloxacin $500 \mathrm{mg}$ twice daily) are indicated where the ulcer is close to the limbus. This may help protect from contiguous spread of infection to the sclera and enhance antibiotic delivery to peripheral lesions. Adjunctive treatment at this stage may include dilating drops and analgesic medication as required.

Subconjunctival antibiotic injections are unpleasant for the patient, introduce a new array of possible complications, ${ }^{20}$ and do not enhance therapeutic efficacy where intensive topical treatment is used. ${ }^{29}$

\section{Initial review}

Early review at 2 days is aimed at detecting rapidly progressive cases. The inflammatory reaction may actually be enhanced with bacterial lysis and endotoxin liberation in the first 48 hours of treatment. Daily review may thus be confusing, and information on which to base any rational modification in treatment (antibiotic sensitivities) would not normally be available until at least 2 days after corneal scraping. Definite progression at this stage (increased 
Indolent and progressive microbial keratitis

Review disease progress

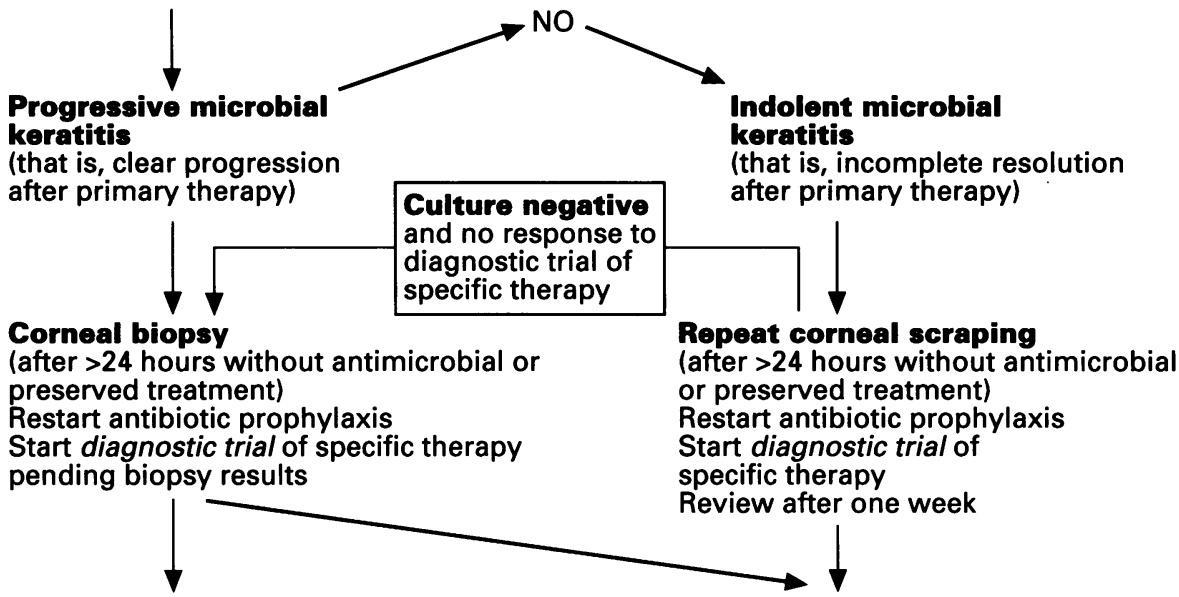

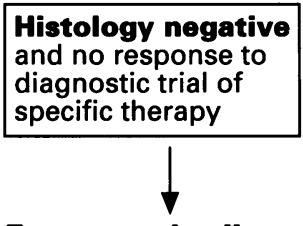

Encourage healing

Maintain antibiotic prophylaxis 4 times a day

Treat any exposure/dry eyes/trichiasis, etc

Use unpreserved medication where possible

Add dexamethasone four times daily

Consider autoimmune melt

Review after 1 week on this regimen where possible

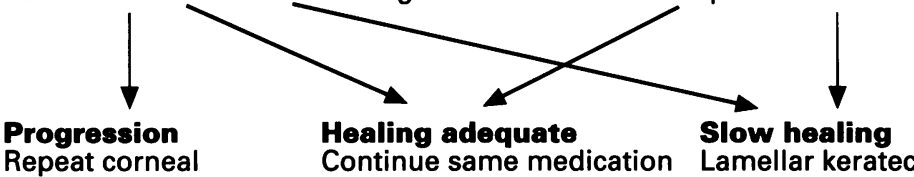

biopsy

Continue same
Review weekly

Trial of systemic

immunosuppression

if still histology

negative

Penetrating

keratoplasty if

progression continues

Figure 5 Some strategies for the further management of indolent and progressive microbial keratitis. The essential priority in the management of these non-urgent referrals is to identify the infecting agent and institute appropriate specific antimicrobial therapy wherever possible. stromal thinning or a clear expansion of the ulcer) is unusual, and implies that patients are either insensitive to, or not complying with, antimicrobial therapy.

Rapid early progression does not permit a pause in antimicrobial therapy with rescraping or corneal biopsy, and undirected changes in antimicrobial chemotherapy at this stage are generally unhelpful. Patients should be admitted to ensure compliance, and the results of initial microscopy, culture, and sensitivity testing should be reviewed. Unless these results indicate possible resistance to primary therapy and a switch to intensive treatment with a more appropriate antibiotic, we would recommend continuing initial broad spectrum antibiotic treatment hourly day and night for 48 hours, then hourly by day for 3 days. Further progression is an indication for specialist referral at this stage.

In contrast with most other cases of microbial keratitis, close clinical monitoring with at least daily review is probably necessary in this small group of patients with rapidly progressive disease. Threatened or actual perforation indicate urgent specialist referral (Fig 3). Emergency penetrating keratoplasties in these circumstances carry a poor prognosis for vision, ${ }^{30}$ are difficult to perform well, and can often be avoided even after a perforation.

\section{Interpretation of culture and sensitivity testing}

While culture specimens should be incubated for a minimum of 14 days before being reported as culture negative, ${ }^{12}$ growth of most pathogens can be expected after 48 hours. ${ }^{11}$ Multiple or confluent colonies growing along the lines of inoculations on agar plates are likely to be genuine pathogenic isolates, particularly when associated with the presence of organisms with the same characteristics on the initial Gram stain. Isolated colonies not growing at the sites of inoculation are likely to be contaminants. Where liquid media are used, contaminants cannot be distinguished from pathogens. Growth in a single broth, without corroboration from solid media, a further broth, or microscopy, should therefore be interpreted with caution.

It is important to appreciate that the commonly used disc diffusion tests of antibiotic sensitivity are based on 
Table 2 Some organisms likely to be involved in progressive and indolent cases of microbial keratitis are tabulated below, along with preferred histological techniques, culture media, and first line antimicrobial therapy. Mycobacteria and anaerobes have been implicated in previous case reports of progressive microbial keratitis, but should be susceptible to initial therapy with ofloxacin

\begin{tabular}{|c|c|c|c|}
\hline Organism & Histology & Culture & Therapy \\
\hline Acanthamoeba $a^{14}$ & $\begin{array}{l}\text { Calcofluor white } \\
\text { Immunofluorescence }\end{array}$ & $\begin{array}{l}\text { Non-nutrient agar } \\
\text { seeded with } E \text { coli }\end{array}$ & $\begin{array}{l}\text { PHMB }(0.02 \%) \\
\text { Propamidine } \\
(0.1 \%)\end{array}$ \\
\hline Fungi ${ }^{13}$ & $\begin{array}{l}\text { Calcofluor white } \\
\text { Gram } \\
\text { Giemsa } \\
\text { PAS }\end{array}$ & $\begin{array}{l}\text { Sabaurauds agar } \\
\text { Brain heart } \\
\text { infusion }\end{array}$ & $\begin{array}{l}\text { Econazole }(1 \%) \\
\text { Itraconazole (100 } \\
\text { mg twice daily) }\end{array}$ \\
\hline $\begin{array}{l}\text { Herpes } \\
\text { simplex }^{50}\end{array}$ & $\begin{array}{l}\text { Electron microscopy } \\
\text { Immunoassay }\end{array}$ & Cell culture & $\begin{array}{l}\text { Acyclovir }(3 \%) \\
\text { Acyclovir }(400 \mathrm{mg} \\
5 \times \text { day })\end{array}$ \\
\hline $\begin{array}{l}\text { Mycobacteria } \\
\text { Anaerobes }^{52} \\
\text { Nocardia }^{53}\end{array}$ & $\begin{array}{l}\text { Ziel-Neelson } \\
\text { Gram } \\
\text { Ziel Neelsen }\end{array}$ & $\begin{array}{l}\text { Lowenstein Jensen } \\
\text { Thioglycolate } \\
\text { Muller Hinton }\end{array}$ & $\begin{array}{l}\text { Ofloxacin }(0 \cdot 3 \%) \\
\text { Ofloxacin }(0.3 \%) \\
\text { Variable }\end{array}$ \\
\hline
\end{tabular}

PHMB = polyhexamethyl biguanide.

antibiotic concentrations achievable in plasma which may be considerably less than those in the inflamed cornea during intensive topical treatment. ${ }^{7}$ Similarly, tube dilution methods, which may be used to quantify bactericidal concentrations for a given isolate, require a degree of qualitative interpretation where the corneal concentration of a drug is uncertain. If clinical progress is satisfactory there is no real indication for altering antibiotic therapy. ${ }^{715}$ Sensitivity testing can be invaluable, however, in guiding the choice of a more appropriate antibiotic where bacterial keratitis is progressive.

\section{Review at 1 week}

Where complete resolution has not occurred at 1 week after presentation, an assessment of whether disease is resolving or progressive is useful in determining a logical pathway for subsequent management (Fig 4). In either case, clear evidence of poor compliance or, in culture positive cases, resistance to the initial antibiotic choice are indications for re-entering the sterilisation phase using appropriate specific therapy. Otherwise, deteriorating or static cases should be referred for specialist management; whereas cases in which resolution is partial but incomplete may safely enter a second phase of treatment directed at encouraging healing.

\section{Phase two - healing}

Healing is commonly retarded in resolving microbial keratitis by treatment toxicity, ${ }^{7}$ untreated underlying ocular surface disease, ${ }^{31}$ or persisting inflammatory activity relating to residual antigens or enzymes released by killed bacteria. ${ }^{32}$ We therefore recommend reducing antibiotic treatment to prophylactic levels, the use of unpreserved medication where possible, and specific attention to dry eyes, exposure, entropion, blepharitis, and other ocular surface disorders during this second phase of initial therapy.

Steroid medication may speed recovery in microbial keratitis but may also enhance fungal or herpetic infection in the absence of specific microbicidal therapy. Complete resolution of anterior chamber and corneal inflammatory signs is normal in microbial keratitis without steroid treatment. Stern and Buttross ${ }^{32}$ in an extensive recent review of the debate surrounding the use of steroid treatment in microbial keratitis concluded that topical steroids should be reserved for cases of culture positive bacterial keratitis in which resolution is incomplete after an initial period of intensive treatment with appropriate microbicidal antibiotics and we recommend that steroid medication is introduced, if appropriate, during phase two of the initial therapy. For microbial keratitis in corneal graft recipients, however, unless there is evidence of fungal infection, steroid therapy should be introduced $\mathrm{ab}$ initio to protect from or treat a concurrent rejection episode.

At review after 1 week of the healing phase (that is, week 3 after presentation), referral may be indicated where healing has not continued to progress. Patients may otherwise simply continue with the healing phase regimen and weekly review until resolution is complete.

\section{Specialist further management of microbial keratitis}

The key elements of further therapy in microbial keratitis are firstly, to identify the infecting organism and modify antimicrobial therapy appropriately, ${ }^{12}$ and secondly, to avoid performing an emergency corneal graft wherever this can safely be delayed until the eye is quiet. ${ }^{30}$ These two principal aims define the therapeutic priorities in the management of progressive keratitis and perforations.

\section{Progressive keratitis}

Progression after 5 days of intensive broad spectrum antibiotic treatment (progressive microbial keratitis), and inadequate healing after an additional week of therapy designed to promote epithelial healing (indolent microbial keratitis), are indications for specialist management. The priority in both these groups is to identify the infecting organism and institute appropriate specific antimicrobial therapy. To improve repeat culture results, all antimicrobial and other preserved medication should be stopped at least 24 hours before specialist review. ${ }^{12}$ In progressive microbial keratitis, corneal biopsy is indicated at this stage. For indolent cases, it is probably reasonable to repeat corneal scraping in the first instance (Fig 5).

\section{Repeat scraping}

At repeat corneal scraping an expanded array of solid and liquid culture media should be inoculated, aiming to accommodate a broad spectrum of possible organisms (Table 2). Ficker et al ${ }^{12}$ have described a comprehensive strategy for microscopy and culture in microbial keratitis. A minimum of two slides should be inoculated, although up to four slides may be helpful to allow a variety of end stains. The order in which solid and liquid culture media are then inoculated will be dictated by clinical suspicion, with media conducive to the culture of the more likely organisms being inoculated first (Table 2).

Suspected early acanthamoebic keratitis is a special case. Acanthamoebae grow well within the corneal epithelium, and where features suggestive of acanthamoebic infection are present (a history of contact lens wear, corneal epitheliopathy, perineural infiltrates, limbitis, and pain), corneal epithelial biopsy is the investigation of choice. ${ }^{14}$ This is performed at the slit-lamp using unpreserved topical anaesthesia. In acanthamoebic keratitis, the corneal epithelium is often only loosely adherent to the underlying stroma, and can usually be debrided easily as an intact sheet. This epithelial specimen is divided, placing one half in fixative (for example, $10 \%$ formal saline) for histological examination, and the other half in normal saline for culture. Subjacent stromal infiltrates may then be scraped and inoculated on slides and culture media as above.

Pending the availability of definitive culture results from repeat scraping, a brief diagnostic trial of specific therapy directed at the most likely isolate, based on the clinical and 
(A)

Excisional biopsy For peripheral lesions

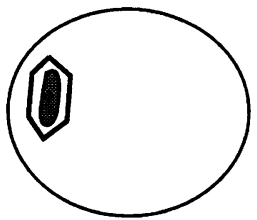

(B)

Incisional biopsy Sparing the visual axis where it is not grossly involved

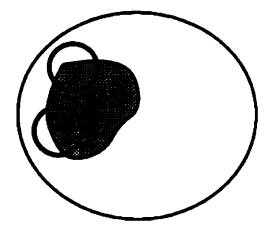

(C)

Lamellar keratectomy Subtotal debridement where the visual axis is grossly involved

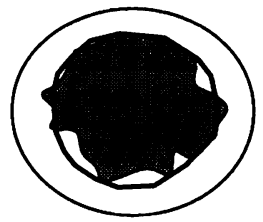

(D) Centrifugal dissection

To minimise the risk of perforation in deep lesions

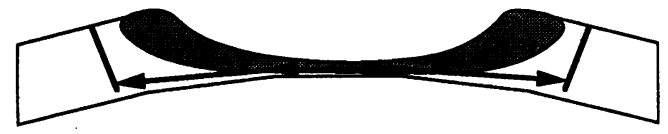

(E) Centripetal dissection

To include all of the ulcer base in shallow lesions

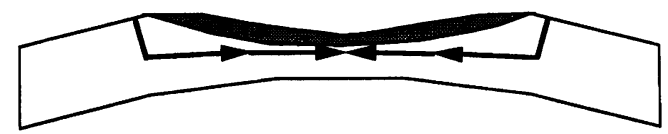

(F) Lamellar flap corneal biopsy

For mid-stromal lesions- for example, crystalline keratopathy

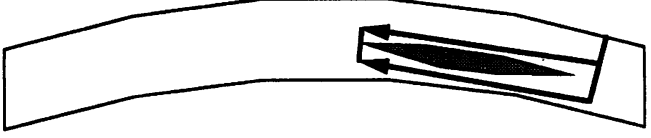

(G) Posterior corneal biopsy

For deep stromal lesions-for example, fungal keratitis

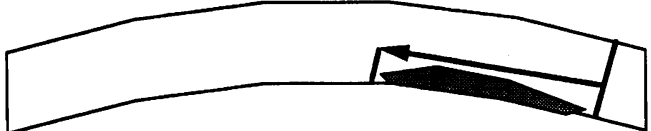

Figure 6 A variety of strategies for corneal biopsy illustrated schematically. To maximise the volume of tissue sampled at the active edge of the lesion, biopsy should ideally include the ulcer base. Smaller peripheral lesions may be excised in total $(A)$. The area to be biopsied is circumscribed using a shouldered diamond blade or trephine, aiming to dissect deep to the ulcer. Where trephines are used to outline incisional biopsies (B), the trephine diameter should be at least $3 \mathrm{~mm}$ to ensure that an adequate tissue specimen is obtained. Multiple incisional biopsies may be taken. For initial biopsies in lesions grossly involving the visual axis, subtotal debridement (C) should aim to include an adequate sample of the lesion's edge while sparing peripheral stromal tissue to facilitate subsequent corneal grafting. Lamellar dissection with a crescent knife is used to lift the biopsy specimen clear. True lamellar dissection is hard to achieve in friable tissue. Commencing lamellar dissection in the thinnest area and working centrifugally (D) may thus be preferable to dissecting in from the groove outlining the lesion where thinning is already pronounced $(E)$. For deep lesions with intact overlying stroma, lamellar ${ }^{39}(F)$ or posterior ${ }^{38}(G)$ corneal biopsy techniques may be appropriate.

epidemiological picture, may be useful in indolent microbial keratitis. A positive clinical response to antifungal or antiviral therapy should be evident within 1 week and may avoid the necessity for corneal biopsy in cases which remain culture negative after repeat scraping. The response to specific therapy is relatively slow in acanthamoebic keratitis, ${ }^{12} 33$ and blind trials of specific therapy are less useful where Acanthamoeba is the suspected isolate. We do not advocate simultaneous use of antimicrobial agents directed against more than one class of organism at this stage, since this may lead to problems with treatment toxicity and diagnostic confusion.

\section{Corneal biopsy}

It is often not possible to culture fastidious or partially treated organisms successfully. Even in the best series, up to $20 \%$ of cases of presumed infective keratitis remain culture negative. ${ }^{1617}$ Where standard scraping techniques have failed, histological examination using light microscopic and immunohistochemical techniques is the logical next step. ${ }^{34} 35$

With careful analysis of serial sections from an adequate biopsy specimen, using a sequential staining strategy, it should be possible to exclude treatable infective corneal pathology with a high level of confidence. ${ }^{35}$ Bacteria, fungi, and protozoa can all be visualised using conventional light microscopic techniques; and immunohistochemical methods may be used to exclude treatable viral infection principally herpes simplex keratitis.

The results of histological analysis are normally available within 48 hours, whereas culture of fastidious organisms may take several weeks. ${ }^{12} 34$ In an experimental comparison of histological examination and culture in corneal biopsy specimens from rabbits with fungal keratitis, histology provided a positive identification of the infecting organism in every case whereas cultures were only positive in $70-80 \%$ depending on the infecting organism. ${ }^{36}$ Positive culture results provide useful additional information, however (for example, antimicrobial sensitivities), and another advantage of corneal biopsy is that enough material is available to inoculate a wide variety of culture media.

Corneal biopsy is the definitive investigation in progressive microbial keratitis. Although irregular astigmatism is a likely sequel to the biopsy of central and larger peripheral lesions, unresolved progressive keratitis has a catalogue of additional complications. An aggressive approach when considering the indications for corneal biopsy is therefore probably justified.

Biopsies should be planned in consultation with pathologists to ensure specimen handling and fixation is optimal, and performed using an operating microscope. Topical anaesthesia is usually adequate. To optimise culture results, anaesthetic drops should be unpreserved and forniceal irrigation during antiseptic preparation should be avoided.

An important secondary role of biopsy is debridement of necrotic tissue. This in itself can be a significant aid to healing. Biopsies should thus aim to excise the lesion in total (excisional biopsy); although the visual axis should be spared where it is not grossly involved (incisional biopsy). A $1 \mathrm{~mm}$ margin of macroscopically uninvolved tissue should be included, where possible, to ensure that the active edge of the ulcer is sampled (Fig 6).

\section{Culture positive indolent or progressive microbial keratitis}

The choice of antimicrobial chemotherapy in culture positive cases of fungal and amoebic keratitis is dictated largely by local clinical experience. In contrast with the initial therapy for bacterial keratitis, sensitivity testing has not yet proved to be of consistent value, prolonged antimicrobial therapy may be required, and sterilisation and healing phases cannot be regarded as distinct.

Acanthamoeba keratitis is becoming increasingly prevalent in London. ${ }^{1433}$ Good results for medical treatment of this condition have recently been obtained using intensive topical combination treatment with polyhexamethyl biguanide (PHMB) and propamidine isethionate (brolene). ${ }^{1433}$ Fungal keratitis is far less common in London. ${ }^{39}$ All recent fungal isolates at Moorfields Eye Hospital have been sensitive to econazole ${ }^{39}$ and first line treatment is currently intensive topical econazole (1\%) with the addition of oral itraconazole $(100 \mathrm{mg}$ twice daily) for peripheral lesions or deep corneal disease.

Histology negative corneal biopsies

A negative corneal biopsy will exclude active microbial keratitis in most cases. However, false negative biopsies 
Threatened or actual perforation

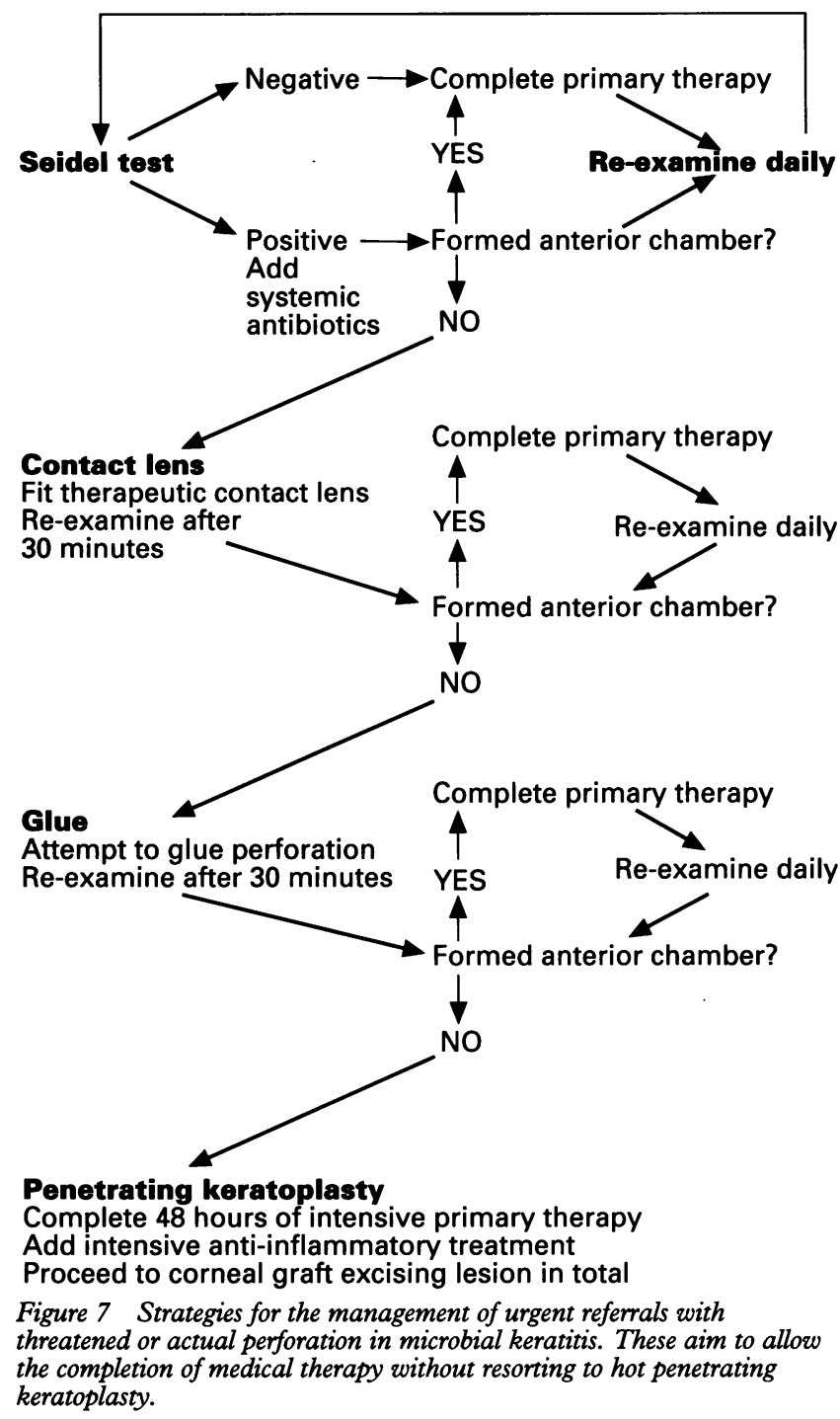

occur. It is important to formulate a strategy that allows for this possibility, but avoids procrastination in the management of histology negative keratitis.

The differential diagnosis at this point lies between unresolved inflammation after effective corneal sterilisation; autoimmune keratitis (for example, rheumatoid disease, Moorens ulcer, etc); other causes of sterile keratitis (ocular surface disease, anaesthesia, exposure, deliberate self harm, etc); and a false negative biopsy. In the absence of clear evidence to the contrary (for example, autoimmune disease), it is probably reasonable to attribute histology negative keratitis to unresolved inflammation. Treatment should enter a healing phase, terminating the diagnostic trial of specific therapy after 5 days, maintaining antibiotic prophylaxis and aiming to optimise the ocular surface environment, with further review after 1 week, or sooner if disease progression has been rapid. Histological examination of biopsy specimens has been shown to exclude fungal infections in particular with a high degree of sensitivity. ${ }^{36}$ It should therefore be safe to add topical steroid medication in most cases after a negative biopsy.

Continued progression after 1 week of therapy directed at promoting resolution of inflammation and epithelial healing suggests either a false negative biopsy, new superinfection despite prophylaxis, or autoimmune keratitis. Patients should be admitted for repeat corneal biopsy. If this repeat biopsy is negative, a trial of intensive systemic immunosuppression may be appropriate. Again fungal infection should have been positively excluded by biopsy.
A

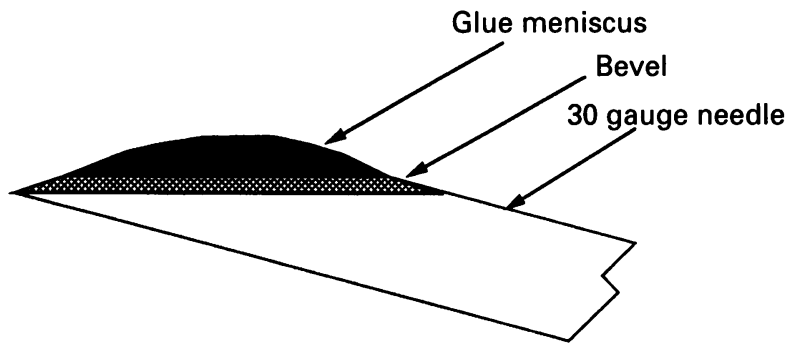

B

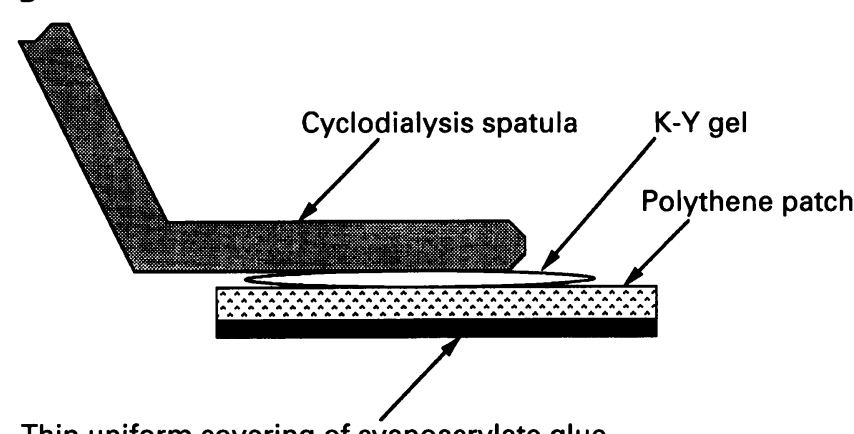

Thin uniform covering of cyanoacrylate glue

Figure 8 (A) A schematic illustration of the slit-lamp biomicroscopic view of the bevel of $a 30$ gauge needle before direct glue application for corneal microperforation $(\leqslant 0 \cdot 25 \mathrm{~mm})$. Only a shallow meniscus of glue should protrude from the bevel, ensuring that a minimal quantity is applied. (B) A schematic illustration of the patch technique of corneal gluing for larger corneal perforations (up to $3 \mathrm{~mm}$ ). A cyclodialysis spatula is dipped in K-Y gel and used to pick up a small polythene patch. The underside of the patch is coated with a thin veneer of glue before application. The quantity of glue applied should be minimal.

Systemic immunosuppression should both produce a clinical response in autoimmune keratitis and improve the prognosis for emergency penetrating keratoplasty in infective keratitis ${ }^{40}$ if this becomes necessary.

For indolent disease, lamellar keratectomy to debride necrotic corneal stroma and temporary central tarsorrhaphy may be considered. If this does not produce rapid healing, early elective penetrating keratoplasty may be indicated for central lesions. ${ }^{41}$ A conjunctival flap may often be a valid alternative for histology negative indolent keratitis in a non-sighted eye or in an elderly patient with good vision in the contralateral eye.

\section{Perforations}

Impending or actual perforation during initial therapy are indications for urgent referral and specialist management (Fig 7). Unless culture and sensitivity results clearly suggest that antimicrobial treatment has been inappropriate, this should be continued for a full 5 day course to complete the sterilisation phase of initial therapy where the anterior chamber remains formed. Broad spectrum systemic antibiotic cover (for example, ciprofloxacin $500 \mathrm{mg}$ twice daily orally) should be added where perforation has occurred.

Microperforations often seal spontaneously, and while the anterior chamber remains deep, no additional intervention is indicated. If the anterior chamber shallows, a therapeutic contact lens may tamponade the leak successfully. If this is going to work, it should do so quickly, and review after 1 hour will determine whether an attempt at corneal gluing is indicated. Only where gluing fails should corneal grafting be considered in an inflamed eye.

\section{Corneal gluing}

Cyanoacrylate glue can be used to seal perforations up to $3 \mathrm{~mm}$ in diameter. ${ }^{42}$ Small perforations may heal beneath the glued area. ${ }^{42} 43$ Importantly though, successful gluing 
buys time, allowing the completion of medical treatment, the resolution of inflammation, and non-urgent penetrating keratoplasty.

A variety of cyanoacrylate analogues have been in ophthalmic use since the early $1960 \mathrm{~s}^{42}$ They are monomers in the liquid form which polymerise rapidly at room temperature after contact with water or a weak base, setting within 1-2 seconds. To reduce toxicity in relation to degradation products, the longer chain cyanoacrylates are preferable, and the quantity of glue applied should be minimised. ${ }^{44}$

The technique used depends on the size of the perforation. For microperforations $(<0.25 \mathrm{~mm})$, a minute quantity of glue (Fig 8A) applied directly with the tip of a 30 gauge needle may suffice. This can be performed at the slit-lamp under topical anaesthesia using a needle to debride the epithelium around the perforation and an absorbent microsurgical sponge to dry the target area immediately before glue application. Iris adherent to a microperforation does not require reposition, since this usually occurs spontaneously once the anterior chamber reforms. Re-examination after 1 hour should reveal complete reformation of the anterior chamber if gluing has been successful.

For larger perforations or those associated with active infection, a patch technique is often required (Fig $8 \mathrm{~B}$ ). Epithelium and necrotic stroma are debrided around the perforation. A small disc of polythene from the nonadhesive portion of a sterile surgical drape, large enough to cover the perforation with a $1 \mathrm{~mm}$ surround, is punched out using a 3-4 mm skin biopsy trephine. This patch is picked up using a cyclodialysis spatula dipped in water soluble gel (for example, K-Y gel - the gel prevents any adhesion between the patch and the spatula). The dry undersurface of the patch is then covered in a thin veneer of cyanoacrylate adhesive applied through a 30 gauge needle beneath the microscope. Immediately after drying the target area, the glue patch is applied. Light pressure is maintained until polymerisation beneath the patch is complete (this is easily visible).

The corneal patch gluing technique can often be performed successfully at the slit-lamp; but an operating theatre environment may be preferable where iris adhesion is extensive. Viscoelastic and air injected through the perforation can be used to reposit adherent iris and minimise aqueous leakage during the procedure. ${ }^{45}$ The amount of viscoelastic used should be minimal, since viscoelastic prolapsing through the perforation may interfere with subsequent adhesion.

Although degradation products may have some antibiotic effect, polymerised glue may also become a focus for infection, and secondary microbial keratitis is a well recognised complication of corneal gluing. ${ }^{42-44}$ Corneal glue may also encourage stromal neovascularisation, increasing the risk of rejection for a subsequent penetrating keratoplasty. So while gluing may be of great value in helping to avoid an emergency graft, a cogent argument can be made for proceeding to early elective glue removal after the resolution of inflammation and, where necessary, penetrating keratoplasty; rather than simply waiting for the glue to detach spontaneously. Short term antibiotic prophylaxis is indicated while the glue remains in situ.

\section{Emergency penetrating keratoplasty}

Relatively good results have been obtained from therapeutic penetrating grafts performed for microbial keratitis with up to $73 \%$ of grafts remaining clear after a variable period of follow up ( 1 month to 9 years). ${ }^{40}$ Hill, comparing early and later grafts for unresolved central infection, found similar results in both groups, ${ }^{41}$ and concluded that earlier penetrating keratoplasty accelerated rehabilitation. However, when emergency grafts in microbial keratitis were compared with a group grafted subsequently for scarring, after complete resolution of infection and inflammation, a clear difference in graft survival was evident, with a $51 \%$ five year survival for emergency grafts versus $90 \%$ for grafts performed in quiet eyes. ${ }^{30}$ Emergency grafts can be technically difficult, and have a high complication rate. ${ }^{304041}$ Inflammation at the time of surgery is a well identified risk factor for graft failure. ${ }^{46}$ Emergency grafts should in general therefore be avoided where possible.

The prognosis for emergency grafting deteriorates for grafts greater than $9 \mathrm{~mm}, 40$ and progressive stromal vascularisation would also significantly increase the risk for graft rejection. ${ }^{4146}$ While emergency grafts should be avoided where possible, early elective penetrating keratoplasty should be considered, before healing is complete, in cases of central indolent or progressive keratitis if the lesion can be encompassed by a standard sized graft.

Failure to seal a perforation with cyanoacrylate adhesive in active keratitis is an absolute indication for penetrating keratoplasty, since irreversible synechial angle closure and secondary glaucoma will result if the anterior chamber remains flat, and there is a significant risk of spontaneous expulsive haemorrhage in the inflamed hypotonous eye. Nevertheless, to minimise the risk of recurrent infection at the graft edge, we advocate at least 48 hours of intensive broad spectrum antibiotic treatment before grafting in patients presenting with a perforation in a previously untreated eye.

In contrast with lamellar keratoplasty, in which recurrence of infection at the graft-host interface is common, ${ }^{41}$ therapeutic penetrating grafts have a high rate of clinical success in eliminating infection where macroscopic excision of the involved area is complete. ${ }^{304041}$ There is thus a strong case for commencing intensive preoperative anti-inflammatory treatment once the decision to perform an emergency graft has been taken. ${ }^{32} 40$ Hourly topical steroids should be added along with prednisolone (60-80 mg) orally. Systemic cyclosporine A may also be indicated where corneal stromal vascularisation is already present or for large eccentric grafts. To modulate the risk of steroid enhancement in progressive fungal keratitis, adjunctive anti-inflammatory treatment may be withheld until 24 hours of intensive antimicrobial treatment have been completed.

Unless the area to be excised at penetrating keratoplasty can easily be contained within a standard graft diameter, a whole fresh eye should be prepared as the donor. ${ }^{30}$ For lesions involving the periphery, one third of the limbus should be spared wherever possible to avoid subsequent graft failure in association with limbal insufficiency. ${ }^{30}$ Fresh donor limbal tissue may be included in the graft where more extensive limbal excision is necessary.

Macroscopically uninvolved tissue adjacent to the lesion may harbour microscopic infection. Urgent postoperative histological examination of the excised specimen is useful to assess whether disease clearance is complete, and to identify or confirm the pathogens involved. ${ }^{30} 40$

\section{Prophylaxis}

An important element in the management of microbial keratitis is the minimisation of risk factors for subsequent infection during or after healing. Nylon corneal sutures should be removed electively and not left to degrade, since broken sutures are a common focus for infection, $47-49$ and any predisposing ocular surface disorder should be treated. 
The position with regard to using antibiotic prophylaxis in the presence of a chronically unstable corneal epithelium (for example, corneal anaesthesia) is not clear. Corneal infection is not uncommon in patients taking topical antibiotics, ${ }^{2}$ and long term prophylaxis may simply encourage selective growth of resistant organisms. ${ }^{47}$ Short term prophylaxis with a non-toxic broad spectrum antibiotic may be indicated in the presence of an epithelial defect, pending resolution or surgical treatment.

\section{Visual rehabilitation}

Stromal opacity or irregular astigmatism may result in a permanent decrease in visual acuity or symptoms attributable to light scatter. Patient satisfaction after corneal grafts performed for uniocular pathology is generally low. ${ }^{46}$ For simple decrements of acuity, with good vision in the fellow eye, contact lens wear or simple reassurance may therefore be preferable to surgical intervention. Where surgical treatment is genuinely indicated once the eye is quiet, lamellar grafting may be considered in the absence of corneal endothelial decompensation.

The Corneal and External Disease Service,

BRUCE D S ALLAN JOHN K G DART

Moorfields Eye Hospital, City Road,

London EC1V 2PD

1 Coster DJ, Badenoch PR. Host, microbial, and pharmacological factors affecting the outcome of suppurative keratitis. $B r \mathcal{F}$ Ophthalmol 1987; 11 96-101.

2 Gudmundsson OS, Ormerod LD, Kenyon KR, Glynn RJ, Baker AS, Haaf $\mathrm{J}$, et al. Factors influencing predilection and outcome in bacterial keratitis. Cornea 1989; 8: 115-21.

3 Dart JKG, Stapleton F, Minassian D. Contact lenses and other risk factors in microbial keratitis. Lancet 1991; 338: 650-3.

4 Schein OD, Buehler PO, Stamler JF, Verdier DD, Katz J. The impact of overnight wear on the risk of contact lens associated ulcerative keratitis. Arch Ophthalmol 1994; 112: 186-90.

5 Bates AK, Morris RJ, Stapleton F, Minassian D, Dart JKG. Sterile corneal infiltrates in contact lens wearers. Eye 1989; 3: 803-10.

6 Stein RM, Clinch TE, Cohen EJ, Genvert GI, Aretensen JJ, Laibson PR. Infected versus sterile corneal infiltrates in contact lens wearers. Am $\mathcal{F}$ Ophthalmol 1988; 105: 632-6.

7 Jones DB. Decision making in the management of microbial keratitis. Ophthalmology 1981; 88: 814-20.

8 Jones DB, Liesegang TJ, Robinson NM. Cumitech 13. Laboratory diagnosis of ocular infections. Washington DC: American Society for Microbiology, 1981.

9 Donnenfield ED, Schrier A, Perry HD, Acclino BS, Gombert ME, Snyder MD. Penetration of topically applied ciprofloxacin norfloxacin and ofloxacin into the aqueous humour. Ophthalmology 1994; 101: 902-5.

10 Dart JKG. Predisposing factors in microbial keratitis: the significance of contact lens wear. Br 7 Ophthalmol 1988; 72: 926-30.

11 Brinser JH, Burd EM. Principles of diagnostic ocular microbiology. In: Tabbara KF, Hyndiuk RA, eds. Infections of the eye. Boston/Toronto: Little Brown, 1986: 73-92.

12 Ficker LA, Kirkness CM, McCartney AC, Seal D. Microbial keratitis - the false negative. Eye 1991; 5: 549-59.

13 Rosa RH, Miller D, Alfonso EC. The changing spectrum of fungal keratitis in south Florida. Ophthalmology 1994; 101: 1005-13.

14 Bacon AS, Dart JKG, Ficker LA, Matheson MM, Wright P. Acanthamoeba keratitis: the value of early diagnosis. Ophthalmology 1993; 100: 1238-43.

15 Baum JL. Initial therapy of suspected microbial ulcers, 1. Broad spectrum therapy based on prevalence of organisms. Surv Ophthalmol 1979; 24: therapy

16 Asbell P, Stenson S. Ulcerative keratitis: survey of thirty years of laboratory experience. Arch Ophthalmol 1982; 100: 77-83.

17 Jones DB. Initial therapy of suspected microbial keratitis II: specific antibiotic therapy based on corneal smears. Surv Ophthalmol 1979; 24: 105-16.

18 Borrmann LR, Leopold IH. The potential use of quinolones in future ocular antimicrobial therapy. Am f Ophthalmol 1988; 106: 227-9.

19 Upadhyay MP, Karmacharya PCD, Koirala S, Tuladhar NR, Bryan LE,
Smolin G, et al. Epidemiologic characteristics, predisposing factors, and etiologic diagnosis of corneal ulceration in Nepal. Am $\mathcal{f}$ Ophthalmol 1991; 111: 92-9.

20 Leibowitz HM. Clinical evaluation of ciprofloxacin $0.3 \%$ ophthalmic solution for the treatment of bacterial keratitis. Am F Ophthalmol 1991; 112 (suppl): $34-47$

21 Wilhelmus KR, Hyndiuk RA, Delmar RC, Abshire RL, Folkens AT, Godio LB. $0 \cdot 3 \%$ Ciprofloxacin in the treatment of bacterial keratitis. Godio LB. 0.3\% Ciprofloxacin in

22 Neu HC. Microbiologic aspects of fluoroquinolones. Am $\mathcal{f}$ Ophthalmol $1991 ; 112$ (suppl): 15-24

23 Lass JH, Mack RJ, Imperia PS, Mallick K, Lazerus HM. An in vitro analysis of aminoglycoside corneal epithelial toxicity. Curr Eye Res 1989; 8: 299-304.

24 Davison CR, Tuft SJ, Dart JKG. Conjunctival necrosis after administration of topical fortified aminoglycosides. Am f Ophthalmol 1991; 111: 690-3.

25 Osato MS, Jensen HG, Trousdale MD, Bosso JA, Borrmann LR, Frank J, Akers P. The comparative in vitro activity of ofloxacin and selected ophthalmic antimicrobial agents against ocular microbial isolates. Am $\mathcal{F}$ Ophthalmol 1989; 108: 380-6.

26 Borrman L, Tang-Liu D, Kann J, Nista J, Lin ET, Frank J. Ofloxacin in human serum, urine and tear film following topical application. Cornea 1992; 11: 226-30.

27 Kanellopoulos AJ, Miller F, Wittpen JR. Deposition of topical ciprofloxacin to prevent re-epithelialisation of a corneal defect. Am f Ophthalmol 1994; 117: 258-9.

28 Groden LR, Brinser JH. Outpatient treatment of microbial corneal ulcers. Arch Ophthalmol 1986; 104: 84-6.

29 Davis SO, Sarff LD, Hyndiuk RA. Comparison of therapeutic routes in experimental pseudomonas keratitis. Am 7 Ophthalmol 1979; 87: 710-6.

30 Kirkness CM, Ficker LA, Steele AD, Rice NS. The role of penetrating keratoplasty in the management of microbial keratitis. Eye 1991; 5: 425-31.

31 Coster DJ. Inflammatory diseases of the outer eye. Trans Ophthalmol Soc UK 1979; 99: 463-79.

32 Stern GA, Buttross $M$. Use of corticosteroids in combination with antimicrobial drugs in the treatment of infectious corneal disease. Ophthalmology 1991; 98: 847-53.

33 Bacon AS, Frazer DG, Dart JKG, Matheson M, Ficker LA, Wright P. A review of seventy two consecutive cases of acanthamoeba keratitis 1984-1992. Eye 1993; 7: 719-25.

34 Newton C, Moore MB, Kaufman HE. Corneal biopsy in chronic keratitis. Arch Ophthalmol 1987; 105: 577-8.

35 Lee P, Green WR. Corneal biopsy. Indications, techniques, and a report of a series of 87 cases. Ophthalmology 1990; 97: 718-21

36 Ishibashi Y, Hommura S, Matsumoto Y. Direct examination vs culture of biopsy specimens for the diagnosis of keratomycosis. Am $f$ Ophthalmol 1987; 103: 636-40.

37 Hwang DG. Lamellar flap corneal biopsy. Ophthalmic Surg 1993; 24: 512-5.

38 Brookes JG, Coster DJ. Non-ulcerative fungal keratitis diagnosed by posterior lamellar biopsy. Aust NZ f Ophthalmol 1993; 21: 115-9.

39 Griffiths MFP, Clayton YM, Dart JKG. Antifungal sensitivity testing of keratitis isolates at Moorfields Eye Hospital 1975-1990: therapeutic implication. In: Bialasiewicz AA, Schaal KP, eds. Infectious diseases of the implication. In: Bialasiewicz AA, Schaal KP, eds.

40 Killingsworth DW, Stern GA, Driebe WT, Knapp A, Dragon DM. Results of therapeutic penetrating keratoplasty. Ophthalmology 1993; 100: $534-41$

41 Hill JC. Use of penetrating keratoplasty in acute bacterial keratitis. Br f Ophthalmol 1986; 70: 502-6.

42 Leahy AB, Gottsch JD, Stark WJ. Clinical experience with N-butyl cyanoacrylate tissue adhesive. Ophthalmology 1993; 100: 173-80.

43 Weiss JL, Williams P, Lindstrom RL, Doughman DJ. The use of tissue adhesives in corneal perforations. Ophthalmology 1983; 90: 610-5.

44 Cavanaugh TB, Gottsch JD. Infectious keratitis and cyanoacrylate adhesive. Am F Ophthalmol 1991; 111: 466-72.

45 Hirst LW. Sodium hyaluronate and tissue adhesive in treating corneal perforations. Ophthalmology 1982; 89: 1250-3.

46 Williams KA, Ash JK, Pararajasegaram P, Harris S, Coster DJ. Long-term outcome after corneal transplantation; visual result and patient perception of success. Ophthalmology 1991; 98: 651-7.

47 Harris DJ, Stulting RD, Waring GO, Wilson LA. Late bacterial and fungal keratitis after corneal transplantation. Ophthalmology 1988; 95: 1450-7.

48 Acheson JF, Lyons CJ. Ocular morbidity due to monofilament nylon corneal sutures. Eye 1991; 5: 106-12.

49 Bates AK, Kirkness CM, Ficker LA, Steele ADMcG, Rice NSC. Microbial keratitis after penetrating keratoplasty. Eye 1990; 4: 74-8.

50 Brik D, Dunkel E, Pavan-Langston D. Herpetic keratitis: persistence of viral particles despite topical and systemic antiviral therapy. Arch Ophthalmol 1993; 111: 522-7.

51 Broadway DC, Kerr-Muir MG, Eykyn SJ, Pambakian H. Mycobacterium cheloniae keratitis: a case report and review of previously reported cases. Eye 1994; 8: 134.

52 Perry LD, Brinser JH, Kolodner H. Anaerobic corneal ulcers. Ophthalmology 1982; 89: 636-42.

53 Hirst LW, Harrison GK, Merz WG, Stark WJ. Nocardia asteroides keratitis. Br f Ophthalmol 1979; 63: 449-54. 